\title{
External validation of Indian diabetes risk score in a rural community of central India
}

\author{
Bharati Taksande $^{1^{*}}$, Minal Ambade ${ }^{2}$, Rajnish Joshi ${ }^{3}$ \\ ${ }^{1}$ Department of Medicine, Jawaharlal Nehru Medical College (JNMC), Sawangi (Meghe), India; \\ ${ }^{*}$ Corresponding Author: bharati.taksande@gmail.com \\ ${ }^{2}$ Mahatma Gandhi Institute of Medical Sciences (MGIMS), Sewagram, India \\ ${ }^{3}$ Department of Medicine, Mahatma Gandhi Institute of Medical Sciences (MGIMS), Sewagram, India
}

Received 12 November 2011; revised 19 December 2011; accepted 30 December 2011

\begin{abstract}
Aim: To find whether the individuals of $\mathbf{4 5}$ years and more of rural area who are in higher tertile of Indian Diabetes Risk Score i.e. of IDRS of $>60$ as compared to those who are in lower tertile i.e. of $<30$, have high frequency of hyperglycemia, impaired glucose tolerance, and manifest diabetes mellitus. Methods: A cross-sectional community based study. The study was conducted in three pre-identified villages. For all consenting and the eligible subjects, the medical student visited their house and the fasting capillary blood glucose was done by One touch blood glucose monitoring system. Four simple questions and one anthropometric measurement for waist circumference helped in deriving the information for Indian Diabetes Risk Score from the same subject. Results: The Indian Diabetes Risk Score (IDRS) (consisting of the factors like age, abdominal obesity, physical inactivity and the family history) which predicted diabetes mellitus in the subject, its sensitivity was $\mathbf{9 7 . 5 0 \%}$ and specificity of $87.89 \%$ when the score of $>I+$ 60 was externally validated on our rural population. Conclusion: Our study demonstrated that the Indian Diabetes Risk Score (IDRS) can be reliably applied as effective tool for the mass screening of diabetes in the community.
\end{abstract}

Keywords: Diabetes Mellitus; IDR; Validation

\section{INTRODUCTION}

Diabetes with its acute attendant and long term complications are a major health hazard today.

In the Indian scenario it has come long way of epidemic and is approaching to the stage of "pandemic". Recently WHO comments that over 19\% of the world's diabetic population currently resides in India [1] Its prevalence in urban India has rose from $2.4 \%$ in 1970 to $15.5 \%$ in 2005 [2]. Insufficient data is available on the prevalence of diabetes in rural India where more than $70 \%$ of our population resides. One study showed that the prevalence was three times higher among the urban (8.2\%) compared to the rural population $(2.4 \%)$ [3] To control the disease, the knowledge of its prevalence and risk factors is necessary in the community. For developing countries, urbanization is considered to be increasing risk factor of diabetes with altered diet, obesity, and decreased physical activity, stress, which differ between urban and rural populations. In developing countries, the majority of people with diabetes are in the 45- to 64-year age range, whereas the prevalence of diabetes in developed countries is more in the age of $>64$ years [4]. The prevalence of diabetes in rural areas was assumed to be one-quarter that of urban areas for Bangladesh, Bhutan, India, the Maldives, Nepal, and Sri Lanka [5]. One of the first studies was the CURE study, which looked at rural and urban communities around city of Chennai. Unfortunately more than $50 \%$ of the diabetic subjects in rural India remain unaware about the disease [6]. There are several potential strategies for diabetes screening the purpose of which is to identify asymptomatic individuals, in whom the screening can modify the course and complications of the disease. Community screening also enhances public awareness of the seriousness of the diabetes. The Indian Diabetes Risk Score (IDRS) was developed by V Mohan and his colleagues and is considered to be one of the strongest predictor of incident diabetes in India [7]. The risk score was derived by conducting the study on a representative sample of Chennai, a large metropolitan city in India, the demography of which is different from that of the rural population. It is a simplified risk score for identifying undiagnosed diabetic subjects using four simple parameters - age, waist circumference, family history of diabetes and physical activity. The individuals were classified as having high risk (score $>60$ ), moderate risk (score $30-50$ ) and low risk (score 
$<30$ ) out of a total score of 100 . IDRS not only predicted diabetes but also predicted metabolic syndrome, even in subjects who had normal glucose tolerance as shown in the recent study [8]. IDRS makes screening programmes more cost effective as it can reduce the cost by $50 \%$ if replaced for screening programmes with blood sugars estimations [6]. A diabetes risk score involving simple non-biochemical measurements, easily applicable simple score could play an important role as the first step in the process of identifying individuals with an increased likelihood of having prevalent but undiagnosed diabetes. However this risk score has not been validated outside the study area where it was developed, and we want to determine if it will remain valid for our rural population of central India as well. Thus current study is planned to externally validate the risk score in the rural population of central India.

\section{AIMS AND OBJECTIVES}

The objective of this study is to find in the individuals of 45 years and more of rural area whether IDRS is more specific and sensitive as a screening tool to diagnose diabetes mellitus when compared with the fasting blood sugar of the subjects.

\section{MATERIAL AND METHODS}

\subsection{Study Design}

A cross-sectional community based study.

\subsection{Settings}

The study was conducted in three villages after the approval from the ethical committee .This villages comprises of 803 people above the age of 45 years. This are pre-identified villages (means identified by the department of medicine where the household number, socioeconomic data and blood pressure were measured for the other project) where the hypertension screening and the follow up measures have already been carried since last year under a community project conducted by the department of Medicine. Under this hypertension project the subjects are already screened for hypertension and obesity. The data regarding various details like the age, gender, history of hypertension, blood pressure, waist and hip circumference of these subjects were already collected.

\subsection{Participants}

A medical student along with the social worker from the department of medicine visited the villages and screened all consecutive subjects: 1) who were more than or equal to 45 years and 2) consented to be the part of this study. We excluded all those: 1) who were unwilling to participate in the study and 2) severely ill subjects and
3) subjects with history of diabetes or taking antidiabetic drugs (oral hypoglycemic agents or insulin).

All the participants were identified by their allotted ID number of 5 digits containing house and person ID (under the hypertension project).

\subsection{Study Procedures}

A medical student obtained the computerized list from the department of Medicine and identified all the people who were more than or equal to 45 years. The details were obtained from the list. Consent was taken from the participants. For all consenting and the eligible subjects, the medical student visited their house and the fasting capillary blood glucose was done by One touch blood glucose monitoring system. To assure the fasting status the social worker informed the subjects to be fasting aday prior to the visit $\mathrm{A}$ fasting blood glucose of more than or equal to $126 \mathrm{mg} \%$ was considered as Diabetes Mellitus. When the fasting blood glucose values were greater than $126 \mathrm{mg} \%$, as per the WHO criteria to see for Impaired Glucose Tolerance, $2 \mathrm{hr}$ post $75 \mathrm{gm}$ of glucose, the venous blood glucose was obtained. 75 gm of anhydrous glucose was dissolved in 1 litre of water and the participants were asked to drink within 5 minutes. Exactly 2 hours after the blood was withdrawn by the trained phlebotomist. The collected samples were sent to the biochemistry laboratory and accordingly labeled as IGT if the postprandial glucose values were $140-200$ $\mathrm{mg} \%$. And those with the glucose values $>200 \mathrm{mg} \%$ were labeled as Diabetic mellitus. The measurement for the waist circumference was taken by the same measuring tape. It was done by keeping the tape at the maximum measurement at the belly. It was measured in centimeters.

Four simple questions and one anthropometric measurement for waist circumference helped in deriving the information for Indian Diabetes Risk Score and the score was obtained from the particulars as shown in Figure 1. These four simple questions are the following:

1) What is your age?

2) Do you have a family history of diabetes? If yes, does your father or mother or both have diabetes?

3) Do you exercise regularly?

4) How physically demanding is your work? [Occupation]

\subsection{Statistical Analysis}

Statistical analysis was done by using Strata SPSS Version 10. We calculated the sensitivity ,specificity, positive predictive values and negative predictive values of IDRS in comparison to fasting blood sugar.

\section{OBSERVATION AND RESULTS}

In the study conducted in a population of 478 people, 


\begin{tabular}{|c|c|}
\hline Particulars & Score \\
\hline \multicolumn{2}{|l|}{ Age (years) } \\
\hline$<35$ & 0 \\
\hline $35-49$ & 20 \\
\hline$>50$ & 30 \\
\hline \multicolumn{2}{|l|}{ Abdominal obesity } \\
\hline Waist $<80 \mathrm{~cm}$ (female),$<90 \mathrm{~cm}$ (male) & 0 \\
\hline Waist $>80-89 \mathrm{~cm}$ (female), $90-99 \mathrm{~cm}$ (male) & 10 \\
\hline Waist $>80-89 \mathrm{~cm}$ (female), $90-99 \mathrm{~cm}$ (male) & 20 \\
\hline \multicolumn{2}{|l|}{ Physical activity } \\
\hline Exercise regular + strenuous work & 0 \\
\hline Exercise regular or strenuous work & 20 \\
\hline No exercise or sedentary work & 30 \\
\hline \multicolumn{2}{|l|}{ Family history } \\
\hline No family history & 0 \\
\hline Either parent & 10 \\
\hline Both parents & 20 \\
\hline Minimum score & 0 \\
\hline Maximum score & 100 \\
\hline \multicolumn{2}{|c|}{$\begin{array}{l}\text { If the score is... } \\
\geq \mathbf{6 0} \text { : Very HIGH RISK of having diabetes. Oral Glucose Toler- } \\
\text { ance Test (OGTT) is recommended to rule out diabetes. If this is } \\
\text { not possible, at least a random blood sugar or a fasting blood sugar } \\
\text { should be done. } \\
\mathbf{3 0}-\mathbf{5 0} \text { : The risk of having diabetes is MODERATE. It is still } \\
\text { recommended to have the above check up. } \\
<30 \text { : Risk of having diabetes is probably LOW. }\end{array}$} \\
\hline
\end{tabular}

Figure 1. Indian Diabetes Risk Score (IDRS)—A simplified Indian Diabetes Risk Score for screening for undiagnosed diabetic subjects developed by V. Mohan et al. [7].

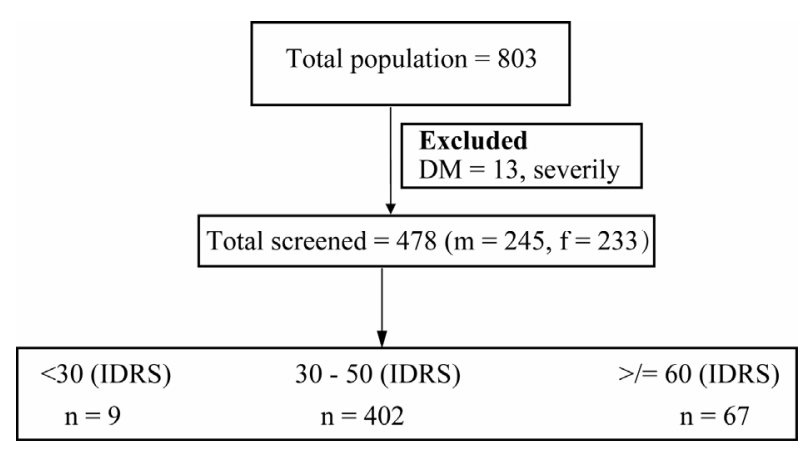

Figure 2. Flow chart of the study.

among which 245 (51.25\%) were males and 233 (48.74\%) females age between 45 to 85 years as shown in Figure 2. The mean age for the population was 56.40 years (S.D.
10.04) (Table 1). Among them, 94 had a history of hypertension which included 39 males and rest females. Out of 478, 9 had an IDRS of 30,402 had an IDRS between 30 - 50 and the rest i.e. 67 had an IDRS of $>60$.

By using strata 10 we calculated the sensitivity, specificity, positive predictive and negative values.

The sensitivity and specificity of IDRS in predicting diabetes mellitus when compared with fasting blood sugars of the subject are given in Table 2 .

The score of $>/=60$ has the $97.50 \%$ sensitivity and $87.89 \%$ specificity and the score of $<30$ and $30-50$ has $100 \%$ and $99.85 \%$ specificity respectively with very low sensitivity as shown in Table 2 .

Thus, the IDRS cannot be easily used to screen the diabetes mellitus or to predict, when the risk score is less than 60 .

\section{DISCUSSION}

Our study showed that the Indian Diabetes Risk Score (IDRS) (consisting of the factors like age, abdominal obesity, physical inactivity and the family history) which predicted diabetes mellitus in the subject, its sensitivity was $92.75 \%$ and specificity of $87.89 \%$ when the score of $>/=60$ was externally validated on our rural population and the score of $<30$ and $30-50$ showed specificity of $100 \%$ and $99.89 \%$ respectively with very low sensitivity. The study by Prabha et al. [9] showed MDRF-IDRS when applied in south Indian population of coastal Karnataka and a score $\geq 60$ is used, by screening $29.7 \%$ (i.e. near $1 / 3$ ) of the population, $62.2 \%$ of the people with undiagnosed diabetes in a population can be detected, with a specificity of $73.7 \%$, which is far less than the results shown in our study. The corresponding figures in the original CURES study by Mohan et al. were $43 \%$ of the population to be screened. $72.5 \%$ sensitivity and $60.1 \%$ specificity for an IDRS score of $\geq 60$. Its ease of administration and its accuracy can help us screen the diabetes in a large population even by a layman since it just involves collection of data on age, family history, physical activity and a single measurement of waist circumference. IDRS could also help to detect people at risk of having prediabetes [8]. Early identification of the high risk individuals would help in taking appropriate intervention in the form of dietary changes and increasing physical activity, thus helping to prevent, or at least delay, the onset of diabetes [7].

There are various diabetes risk score but to our knowledge this is the simplest of all risk score. Ramachandran et al. [10] from South India have also developed a Diabetes Risk.

Score which was validated in three cohorts by using one of the different scoring system which included BMI. A score of $>21$ gave a sensitivity, specificity,positive 
Table 1. Baseline characteristics of the population surveyed.

\begin{tabular}{|c|c|c|c|c|c|}
\hline Variables & Observation & Mean & Std deviation & Min & Max \\
\hline Age (years) & 478 & 56.40 & 10.04 & 45 & 85 \\
\hline Smoking yrs & 478 & .13 & 0.33 & 0 & 1 \\
\hline Tobacco yrs & 478 & 12.93 & 18.56 & 0 & 73 \\
\hline Alcohol yrs & 478 & 1.55 & 7.29 & 0 & 60 \\
\hline SBP_average & 478 & 129.43 & 21.24 & 75 & 223 \\
\hline DBP_average & 478 & 81.27 & 11.50 & 50 & 128.33 \\
\hline Height (cm) & 478 & 155.89 & 10.08 & 121 & 189 \\
\hline Weight (kg) & 478 & 49.88 & 10.45 & 27 & 87 \\
\hline Waist Hip Ratio (WHR) & 478 & .86 & 0.07 & 0.57 & 1.24 \\
\hline Sugar (mg\%) & 478 & 102.57 & 30.98 & 64 & 521 \\
\hline BMI (meter $/ \mathbf{k g}^{2}$ ) & 478 & 20.48 & 3.71 & 12.32 & 38.66 \\
\hline
\end{tabular}

Table 2. Sensitivity and Specificity of IDRS.

\begin{tabular}{cccc}
\hline IDRS & $<\mathbf{3 0}$ & $\mathbf{3 0 - 5 0}$ & $>/=\mathbf{6 0}$ \\
\hline Sensitivity & $52.65 \%$ & $5.47 \%$ & $97.50 \%$ \\
specificity & $100 \%$ & $99.85 \%$ & $87.89 \%$ \\
PPV & $100 \%$ & $99.61 \%$ & $23.16 \%$ \\
NPV & $52.65 \%$ & $46.92 \%$ & $99.95 \%$ \\
\hline
\end{tabular}

predictive value and negative predictive value of $76.6 \%$, $59.9 \%, 9.4 \%$ and $97.9 \%$ in cohort $1,72.4,59 \%, 8.3 \%$ and $97.6 \%$ in cohort 2 and $73.7 \%, 61.0 \%, 12.2 \%$ and $96 \%$ in cohort 3 . The disadvantage of this risk score was that it increased both the time and the cost for screening as BMI and waist were calculated.

Thus this simplified Indian Diabetes Risk Score developed by V Mohan et al where only single waist measurement and simple 3 questions is included is one of the best diabetes risk score with very good sensitivity and specificity and useful tool to predict and screen undiagnosed diabetes mellitus in the population. Our study validates its sensitivity and specificity in the rural population of central India. Our study had the limitation that the study population was very small .For external validation the population has to be large. Our study implies that the IDRS can be the easiest applicable score to predict diabetes mellitus in the population but the sensitivity and specificity of this score increases only when the IDRS > 60.

\section{CONCLUSION}

The results of our study demonstrate that the Indian Diabetes Risk Score (IDRS) though being simple, fast and cost effective tool cannot be reliably applied as effective tool for the mass screening of diabetes in the community and therefore can only up to certain extent help in identifying undiagnosed from the population.

\section{ACKNOWLEDGEMENTS}

We highly acknowledge the community health workers of that villages who helped us for the screening survey of the subjects.

\section{REFERENCES}

[1] Wild, S., Roglic, G., Green, A., et al. (2004) Global prevalence of diabetes, estimates for the year 2000 and projections for 2030. Diabetes Care, 27, 1047-1053. doi: $10.2337 /$ diacare.27.5.1047

[2] Thomas, R. (2009) The Hindu. Online Edition of India's National Newspaper. 17 October.

[3] Ramachandran, A., Snehalatha, C., Dharmaraj, D. and Viswanathan, M. (1992) Prevalence of glucose al intolerance in Asian Indians. Urban-rural difference and significance of upper body adiposity. Diabetes Care, $\mathbf{1 5}$ 1348-1355. doi:10.2337/diacare.15.10.1348

[4] King, H., Aubert, R.E. and Herman, W.H. (1998) Global burden of diabetes, 1995-2025: Prevalence, numerical estimates, and projections. Diabetes Care, 21, 1414-1431. doi:10.2337/diacare.21.9.1414

[5] Ramachandran, A., Snehalatha, C., Latha, E., Manoharan, M. and Vijay, V. (1992) Impacts of urbanization on the lifestyle and on the prevalence of diabetes in native Asian Indian population. Diabetes Research and Clinical Practice, 44, 207-213. doi:10.1016/S0168-8227(99)00024-8

[6] Deepa, M., Deepa, R., Shanthirani, C.S., Datta, M., Unwin, N.C., Kapur, A. and Mohan, V. (2005) Awareness and knowledge of diabetes in Chennai-The Chennai Urban Rural Epidemiology Study (CURES-9). Journal of 
the Association of Physicians India, 53, 283-287.

[7] Mohan, V., Deepa, R., Deepa, M., Somannavar, S. and Datta, M. (2005) A simplified Indian diabetes risk score for screening for undiagnosed diabeteic subjects. Journal of the Association of Physicians of India, 53,759-763.

[8] Mohan, V., Sandeep, S., Deepa, M., Gokulakrishnan, K., Datta, M. and Deepa, R. (2007) A diabetes risk score helps identify metabolic syndromeand cardiovascular risk in Indians - The Chennai urban rural epidemiology study (CURES-40). Diabetes, Obesity and Metabolism, 9, 337-
343. doi:10.1111/j.1463-1326.2006.00612.x

[9] Adhikari, P., Pathak, R. and Kotian, S. (2010) IDRS in another South Indian population through the boloor diabetes study (BDS). Journal of the Association of Physicians of India, 58, 434-436.

[10] Ramachandran, A., Snehalatha, C., Vijay, V., Wareham, N.J. and Colagiuri, S. (2005) Derivation and validation of diabetes risk score for urban Asian. Diabetes Research and Clinical Practice, 70, 63-70.

doi:10.1016/j.diabres.2005.02.016 